\title{
Eumycetoma: present status and perspective
}

\author{
Roberto Arenas ${ }^{1}$, Ma. Elisa Vega-Mémije² and Lucía Rangel-Gamboa*

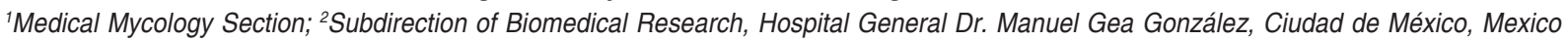

\begin{abstract}
The eumycetoma is a severely debilitating chronic progressive fungal cutaneous infection. Classic clinical triad is characterized by painless subcutaneous mass, sinus tracts formation and sero-purulent discharge that contain aggregates of fungal hyphae called grains. Any part of the body can have affected, with extension to muscular or bone, even visceral compromised. The eumycetoma is observed in tropical and subtropical countries; In Latin-America, is reported with less frequency. In endemic areas, antibody presence again etiological agents were higher compared with number of people affected, thus it is supposed that individual genetic susceptibility most by exist. Recently, it was reported specific polymorphism in genes CR1, IL-8, NOS2 and chitriosidase, which were associated with development of eumycetoma. The diagnosis is suggested by clinical presentation; the histopathology and microbiology studies, plus radiologic valuation confirmed diagnosis. Madurella mycetomatis is the most informed etiological agent. Using phylogenetic tools new species in genus Madurella were reported; moreover, Trematosphaeria grisea and Pseudallescheria boydii were reclassified. Etiological agent Identification is important, because differences in antifungal susceptibility exist. Eumycetoma treatment includes surgery plus antifungal drugs. Identification of etiological agents is primordial, because antifungal resistance could exist. To development new pharmacological strategies, comprehension of grain formation physiology and drugs effects are necessary.
\end{abstract}

KEY WORDS: Eumycetoma. Madura foot. Madurella. Trematosphaeria. Pseudallescheria.

\section{Introduction}

Mycetoma is a subcutaneous, granulomatous-type, chronic infectious disease that is endemic in tropical and subtropical areas, which can be caused by more than 56 different microorganisms, including bacteria, then being called actinomycetoma, and fungi, then being called eumycetoma'. In this review we will focus on eumycetoma. The characteristic clinical triad is comprised by not-too-painful volume increase, formation of fistulae and purulent or seropurulent exudate containing "grains". It can extend and compromise deep structures, which results in destruction, deformity and loss of function, sometimes even being fatal. The foot is the most commonly affected topology, accounting for $82 \%$ of cases (Fig. 1 and 2). It can occur in other localizations, such as the knees, arms and legs, head and neck, and even the glutei and the perineum. It is more common in young, male adults, between 20 and 40 years of age. Children can also be affected in up to $30 \%$ of cases ${ }^{2}$. Many patients are low socioeconomic peasants or laborers. A distinctive characteristic of mycetoma is the formation of the parasitic elements called "grains", which are constituted by microorganisms, probably as part of a defense mechanism against the host immune system ${ }^{3}$. Given that a diversity of microorganisms can produce the disease, there is great morphologic variation found in the characteristics of the grains, which are related to the causative agent.

\section{Method}

A search was conducted in indexed international journals using the PubMed database (http://www.ncbi.

\section{Correspondence:}

Lucía Rangel-Gamboa

Calzada de Tlalpan, 4800

C.P. 14080, Ciudad de México, México

E-mail: draluciarangel@yahoo.com.mx
Date of reception: 05-10-2016

Date of acceptance: 06-12-2016

DOI://dx.doi.org/10.24875/GMM.M18000091
Gac Med Mex. 2017;153:763-772

Contents available at PubMed www.gacetamedicademexico.com 
nlm.nih.gov/pubmed) and the word "Eumycetoma" including articles published from January 1, 2000 to June 15, 2016, also relevant articles related to specific subjects that did not appear in the search being additionally included.

\section{Objective}

To analyze recent information in such a way that enabled a full review of the subject, including epidemiological, clinical, diagnostic and therapeutic aspects, research models and insights of interests for general practitioners, dermatologists and mycologists.

\section{Results}

Fifty-six articles were found, out of which a summary of the most relevant findings is next presented, and 16 articles containing associated and notable information are additionally cited.

\section{Historical data}

The term "mycetoma" stems from Greek mykes (fungus) and oma (tumor). In India, Athatva Veda refers it as pada valmikan, which means "foot anthill"; however, the first official description of mycetoma, in 1842, is attributed to Dr. John Gill, who reported the "Madura foot" in India. Subsequently, Colebrook, from the same public health center, described more cases and referred to it as a distinctive disease of the region. Between 1860 and 1874, Vandyke Carter mentioned initially the damage caused both to soft tissue and bone, and subsequently detailed the presence of fungal structures in grains obtained from the affected area. At early $20^{\text {th }}$ century, Pinoy classified the cases according to the causative microorganism ${ }^{5}$. In Mexico, in 1963, Latapi identified the grains in human eumycetomas, originally described in cattle actinomycosis by Bollinger in the $19^{\text {th }}$ century ${ }^{6}$.

\section{Epidemiology}

Eumycetoma has a global distribution; it is found in arid zones with short rain seasons, in countries with tropical and subtropical climate located between the Cancer and Capricorn tropics, in the zone known as the mycetoma belt, and it predominates in the west of Africa, between Senegal and Sierra Leona, with Sudan standing out as endemic area ${ }^{7}$ In the Asiatic continent is reported more frequently in India and Yemen ${ }^{8}$, while in Latin America its frequency is lower compared with actinomycetoma and occurs mainly in Mexico, Guatemala and Venezuela ${ }^{9-11}$. In Mexico, eumycetomas account for $3.48 \%$ to $7.88 \%$ and actinomycetomas for $96.52 \%$ to $92.12 \%{ }^{12,13}$. In a review of 3933 cases of mycetoma in the Mexican Republic, which corresponded to an incidence of 73 new cases per year, 137 eumycetomas were found, with Madurella grisea $(n=39)$ being observed to have a slightly higher frequency than Madurella mycetomatis $(n=36)$, which is the most common causative agent in the world ${ }^{13}$. In Europe and Israel, the migration processes of population native to endemic areas have increased the number of reported cases in the past few years ${ }^{14,15}$. In endemic areas, it occurs more commonly in people of low socioeconomic stratus who walk barefoot or wearing open shoes. In these references, mycetoma predominated in the male gender at a 4:1 ratio, probably for being more exposed to working activities such as agriculture. The disease is rare in children, but in endemic countries such as Sudan, numerous cases in children have been report$\mathrm{ed}^{2}$. Although eumycetoma is predominant in humans, is not exclusive of them and has been reported in other species such as dogs, horses and even buffaloes ${ }^{16}$.

\section{Clinical presentation}

The described condition occurs in the skin and subcutaneous cellular tissue, but it can also compromise muscle fascia, tendons, muscles and bones. The lower limbs (Fig. 1), followed by the upper limbs, are the most affected regions; however, it has been reported in the trunk, abdominal wall, the jaw, paranasal sinuses, the orbit, the eyelids, the skull and the central nervous system, the vulva, the scrotum and surgical incisions ${ }^{17-19}$. The lesions are characterized by volume increase, which may be accompanied by the presence of fistulae and seropurulent exudate, with a slow and progressive evolution over months or years ${ }^{20}$. In suppurative lesions, grains can be observed at plain sight. The lesions can exacerbate during pregnancy owing to the immunosuppression that accompanies gestation, as well as to the effect of estrogen on fungi, which possess hormone receptors on their membrane $^{21}$. In the absence of diagnosis and appropriate treatment, eumycetomas produce significant morbidity secondary to disease progression, which can generate the following complications: bone destruction and deformity, lymphatic obstruction, secondary bacterial infection, pathologic fractures, nerve compression and paralysis, osteopenia and osteoporosis. 

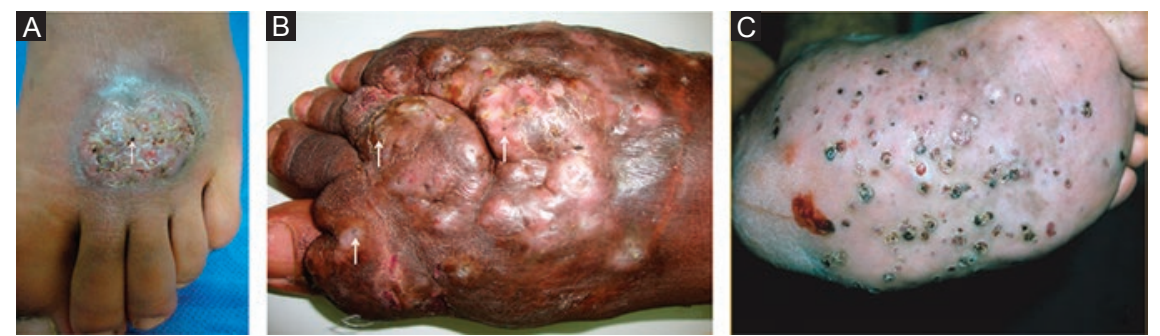

Figure 1. Clinical images. Increased volume, fistulae formation and exudate is observed in all lesions. A and B: arrows pointing at the black grains. C: easily visible multiple grains. B and C: longstanding lesion, where increased volume and fibrosis cause deformity.

\section{Pathophysiology}

The causative agents are found in the environment, including the soil, and many people are therefore exposed. However, most people don't develop mycetoma, since introduction of contaminated material is necessary by means of trauma; at Sudan endemic areas, acacia thorns have been associated with the development of the disease ${ }^{22}$. The inoculum amount, the host immune response and the hormone environment are factors regarded as important for the progression of the disease. Once the infectious agent penetrates, facilitated by a minor trauma, it organizes itself by forming grains inside the host. The grain consists of a dense package of fungal micelles imbibed in a material with cement properties of brown to black color ${ }^{8}$. The details of the chemical are not known, but it is known to contain lipids, proteins, DHN-type melanin, copper, zinc and calcium. The immune system tries to eliminate the grains by producing cytokine and enzymes, including chitinases. Both AMCase chitinase and chitotriosidase have been shown to bind to the chitin present in it. A polymorphism in the chitotriosidase gene was identified, the phenotype of which is manifested in enzyme inactivity, and it was associated with a higher risk of developing mycetoma with $M$. mycetomatis ${ }^{23}$, which suggests susceptibility of the host. Furthermore, the efficient participation of the immune system was supported with epidemiological studies in endemic areas, where up to $80 \%$ individuals were reported to have antibodies, with the number of individuals who develop the disease being comparatively low. Evidence indicates that patients who develop the disease exhibit innate immunity failure ${ }^{24,25}$. Several genes involved in neutrophil function were studied in patients infected with $M$. mycetomatis, with Hardy-Weinberg unbalance being found for single-nucleotide polymorphisms (SNP), in complement receptor 1 , interleukin 8 (IL-8) and type 2 nitric oxide synthase (NOS2), which are correlated with IL-8 higher expression and NOS2 low secretion, whereas SNPs that induce IL-8 low expression and NOS2 high production were found in healthy controls ${ }^{26}$. In general, cell immune response is important to eliminate fungal infections. Nasr et al. ${ }^{27}$ demonstrated Th-1-type cellular immunity in patients with eumycetoma by M. mycetomatis, who had increased levels of Th-1-type cytokines (interferon gamma, tumor necrosis factor alpha, IL-1 $\beta$ and IL-2) in comparison with healthy controls.

\section{Diagnosis}

Clinical presentation is important for diagnosis and, since it can be difficult, especially in initial lesions with few clinical data (mini-mycetoma), both mycology and imaging studies are indispensable. In longstanding lesions, grains can be commonly be observed at plain sight when smear of a fistula purulent exudate is obtained. Grains can be black in color if caused by M. mycetomatis, Trematosphaeria grisea, Falciformispora senegalensis (formerly Leptosphaeria senegalensis), Falciformispora thompkinsii, Exophiala jeanselmei, Pyrenochaeta romeroi, Curvularia lunata and Phialophora verrucosa, among other agents; or white if caused by Scedosporium apiospermum, Aspergillus nidulans, Aspergillus flavus, Fusarium sp., Acremonium sp., Nostestudina rosatti and Microsporum audouinii, among the most common ${ }^{6}$. Actinomycetomas can produce grains, usually of red or yellow color, but they can produce white grains as well, and microbiological is therefore essential to differentiate between eumycetoma and actinomycetoma ${ }^{28}$. To verify the presence of the causative agent, direct examination should be carried out, as well as purulent exudate sampling for culture. The material obtained from the lesion is placed on a microscope slide, a drop of Lugol's iodine solution, saline or $10 \%$ potassium hydroxide is added, and the slide cover is placed. The grains' characteristics can guide the etiologic diagnosis. In case there are fistulae, the culture can be made with the obtained secretions; otherwise, the material is obtained by 
Table 1. Current taxonomic classification of the most common eumycetoma-causative species

\begin{tabular}{|c|c|c|c|c|c|c|c|}
\hline Division & Subdivision & Class & Subclase & Order & Family & Genus & Species \\
\hline \multirow[t]{3}{*}{ Ascomycota } & Pezizomycotina & Sordariomycetes & Sordariomycetidae & Sordariales & & Madurella & $\begin{array}{l}\text { mycetomatis } \\
\text { pseudomycetomatis } \\
\text { fahalii }\end{array}$ \\
\hline & & Dothideomycetes & Pleosporomycetidae & Pleosporales & Melanommataceae & Trematosphaeria & grisea \\
\hline & & Sordariomycetes & Hypocreomycetidae & Microascales & Microascaceae & $\begin{array}{l}\text { Pseudallescheria } \\
\text { Scedosporium }\end{array}$ & $\begin{array}{l}\text { boydii } \\
\text { apiospermun }\end{array}$ \\
\hline
\end{tabular}

Information obtained from Mycobank: http://www.mycobank.org/quicksearch.aspx

aspiration in closed lesions (cryptic eumycetomas) or from tissues obtained by incisional biopsy. Two temperatures should be used for culture growth: $26^{\circ} \mathrm{C}$ to rule out dermatophytes, which are rather uncommon eumycetoma causative agents, and $37^{\circ} \mathrm{C}$ to enable isolation of most human pathogens. Cultures should be preserved for up to 6 weeks, since some agents, such as $M$. mycetomatis, grow quite slowly. Culture in media for bacteria is indicated in order to establish the differential diagnosis with actinomycetoma ${ }^{6}$. In spite of all cautions, identifying the causative agent genus and species is not always possible, since cultures can be contaminated with bacteria or be negative. When the culture is negative, it should be assessed by an expert, since fungal structures identification is complex ${ }^{1,2,9}$.

Characteristics of the grains and cultures of the most commonly reported species in the entire world are described next.

\section{Madurella mycetomatis}

It is the most widely reported species. On direct macroscopic examination, grains are black, globular or lobulated, from 0.5 to $5 \mathrm{~mm}$, and one or more can be observed. On microscope, the grains are reddish brown in color, and the hyphae that make them up appear in color brown. On culture macroscopic observation, the colony is greyish, membranous, and subsequently it can be observed of yellow-brown color with dark pigmentation at the center. It grows at $26^{\circ} \mathrm{C}$, and optimally at $30^{\circ} \mathrm{C}$. Microscopically, it exhibits 3.5 to $5 \mu \mathrm{m}$ pyriform conidia, which originate from simple or branched conidiophore fungi. In corn flour medium, it can develop microconidia ${ }^{29}$.

\section{Madurella pseudomycetomatis, Madurella tropica and Madurella fahalii}

These recently-described species should be differentiated from M. mycetomatis. These are dematiaceous fungi that fail to produce conidia or ascospores and, by definition, belong to the Madurella genus. They produce greyish-brown color colonies; on the back, orange, brown or grey pigmentation is observed. Microscopically, pigmented, sterile septate hyphae that can produce chlamydospores are appreciated. They have optimal growth at $30^{\circ} \mathrm{C}$. There are no known teleomorph forms. Madurella fahalii is differentiated for not presenting diffusible pigment. These species are identified by molecular biology using the ITS sequence, the beta tubulin gene and the RNA polymerase II subunit gene ${ }^{30}$.

\section{Trematosphaeria grisea (formerly Madurella grisea) (Table 1)}

In the patient, it appears with black, globular or lobulated grains, of 0.5 to $1 \mathrm{~mm}$ in diameter, which can initially be soft and subsequently rock-hard. When cultured, the colonies exhibit optimal growth at 27-30 ${ }^{\circ} \mathrm{C}$ (maximum $40{ }^{\circ} \mathrm{C}$ ), and can develop reddish-brown pigmentation (Fig. 2 a and b). Microscopically, hyaline or brown septate hyphae, of 1 to $3 \mu \mathrm{m}$, with thick walls, are observed. The conidia measure 2-4 $\mu \mathrm{m}$. Conidiophores are hyaline and short. Environmental isolates can develop pycnidia (asexual fruiting bodies) after 8 weeks' incubation, which are observed as small black dots with white droplets on top of the culture. The sexual form is unknown ${ }^{31}$.

\section{Pseudallescheria boydii/Scedosporium apiospermum complex}

Until recently, Pseudallescheria boydii and Scedosporium apiospermum were regarded as the teleomorph (asexual) and anamorph (asexual) states, respectively, of the same fungus, but currently they are reclassified as different species: $S$. apiospermum, the heterothallic teleomorph form of which is called Pseudallescheria apiosperma, whereas Scedosporium boydii homothallic teleomorph form is Pseudallescheria 

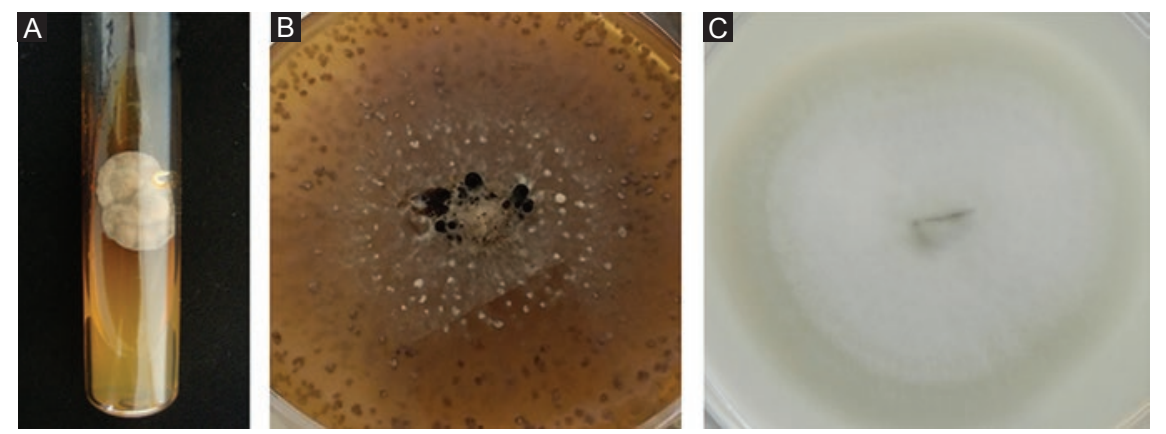

Figure 2. Cultures, A and B: Trematosphaeria grisea. B: incipient sclerotia are observed. C: Pseudallescheria boydii complex.
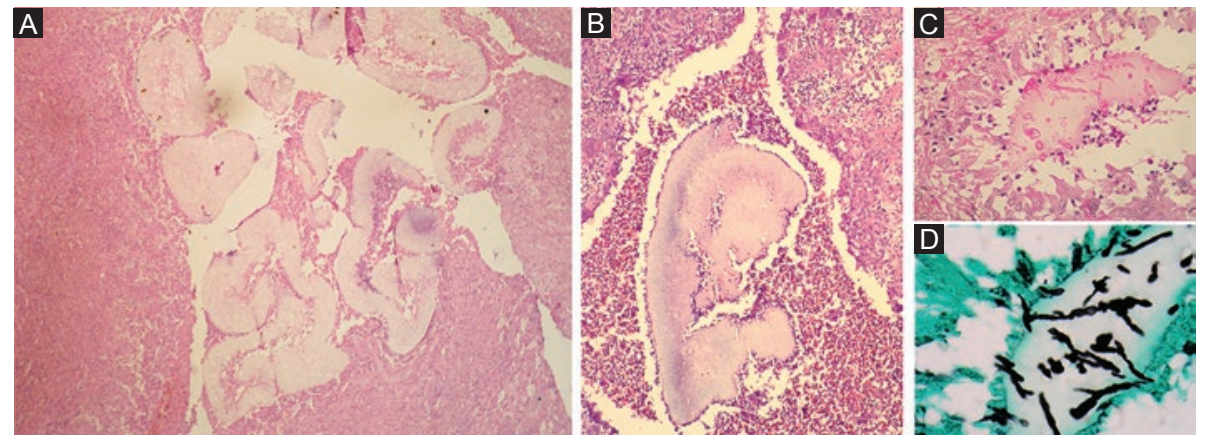

Figure 3. Histologic images of a grain produced by P. boydii complex. A: H\&E staining, where a multilobulated grain is observed (10x). B: inflammatory infiltrate and fistulae formation stand out. C and D: PAS and Gomori Grocott special stainings (respectively), where hyphae are observed inside the grain.

boydii (Table 1). For differentiation, ITS region sequences, the beta tubulin gene and calmodulin are required ${ }^{32,33}$.

The eumycetoma-causative agent most commonly reported in Europe is $S$. apiospermum, especially in immunosuppressed subjects ${ }^{14}$. In infected patients, fungi of this complex produce yellowish-white, lobulated or globular grains of 1 to $2 \mathrm{~mm}$ in diameter. It grows quickly in Sabouraud-glucose agar at $25^{\circ} \mathrm{C}$, but it can tolerate $37^{\circ} \mathrm{C}$ and even up to $42^{\circ} \mathrm{C}$. Initially white colonies turn dark grey or smoked brown color in the surface, while the back is observed with brownish grey or black areas. Microscopically, it exhibits hyaline, flexuous hyphae of 2 to $5 \mu \mathrm{m}$, and brown color-pigmented conidia. The teleomorph forms develop in corn flour agar or dextrose-potato agar, and show oval or globular asci containing eight ascospores. When the ascus wall breaks, it releases elliptic, dark ascospores of 4 to $8 \mu \mathrm{m}$ in diameter ${ }^{31-33}$.

\section{Histopathological diagnosis}

Anatomopathological study is considered to be the next step when direct examination fails ${ }^{6}$. Characteristically, grains are observed surrounded by a suppurative-type granuloma, located in the dermis or in subcutaneous cellular tissue. Around the suppuration zone, there is a palisade of histiocytes; outside this palisade, a mixed inflammatory infiltrate composed of neutrophils, plasmatic cells and histiocytes can be observed, accompanied by progressive fibrosis. Special staining, such as Gomori Grocott, periodic acid-Schiff (PAS) stain and Gram staining, can help to distinguish the grain varieties. In eumycetomas, large, segmented filaments of 2 to $4 \mu \mathrm{m}$ in diameter, with rod-shaped, bulgy hyphae and chlamydospores can be observed (Fig. 3 and 4); this image contrasts with that of actinomycetomas, where thin, gram-positive filaments of $1 \mu \mathrm{m}$ or less in diameter are revealed. In the specific case of M. mycetomatis, 0.5 to $3-\mathrm{mm}$, round, oval or trilobulated grains are described, which are composed of a central zone and a more compact external layer. Two filamentous and vesicular types of grain are described. M. mycetomatis grains stained with hematoxylin-eosin (H\&E) contain a homogeneous pink material (cement-type), while the hyphae are observed in the periphery. With the Fontana-Mason staining, the presence of melanin is demonstrated at the grain periphery and hyphae, which acquire a blackish-brown color (Fig. 5). The presence in the grain structure of heavy metals, proteins and lipids, 

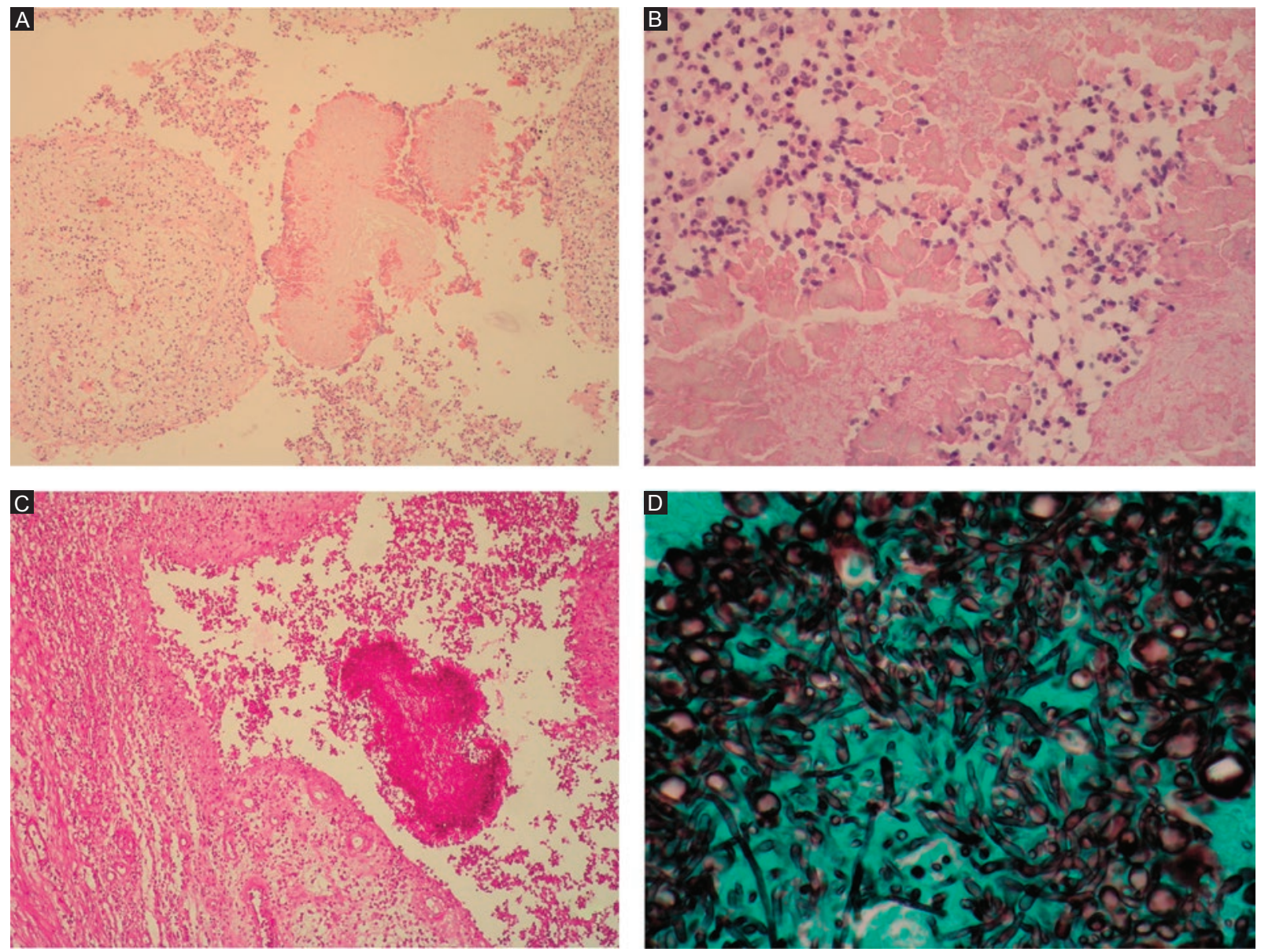

Figure 4. Histologic images of a grain produced by Acremonium sp. A: H\&E staining, where a grain surrounded by inflammatory infiltrate and fistula formation is observed (10x). B: at higher augmentation, neutrophils in the inflammatory infiltrate stand out (40x). C: PAS staining (10x). D: PAS staining, where hyphae are observed inside the grain (40x).

which are elements that presumably hinder antifungal agents penetration, has also been demonstrated ${ }^{34,35}$.

\section{Radiological diagnosis}

Imaging techniques are used to determine the extent of the lesions since, as previously mentioned, eumycetoma can affect deep tissues and even the bones (bone involvement should be ruled out in especially longstanding lesions). Standard radiography can be useful to define lesion limits and whether there is bone involvement ${ }^{36}$. Abd El Bagi ${ }^{37}$ proposed a radiological classification to define the degree of compromise according to alterations found in radiological images; it goes from stage 0 , where the lesion is limited to the penetration site, with soft tissue inflammation and without bone compromise, to stage $\mathrm{VI}$, which shows multidirectional dissemination with complete destruction of bone structures. However, radiological changes are only evident in advanced disease,

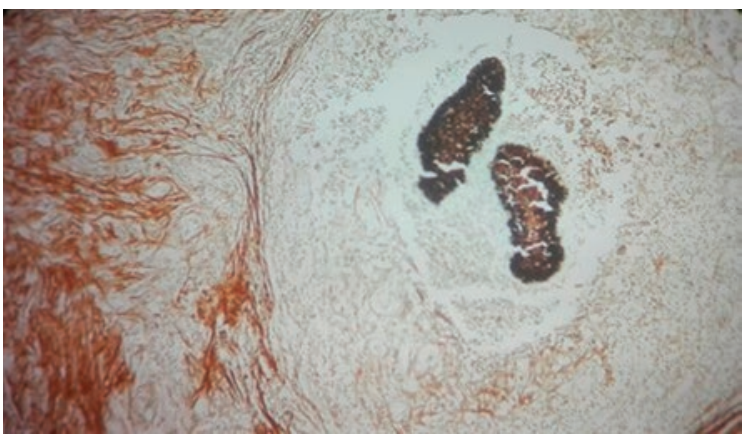

Figure 5. M. mycetomatis grains stained with Fontana-Masson staining show melanin at their periphery, which exhibits a blackish-brown color.

and adequately determining soft tissue compromise is not possible with plain radiograph; in addition, imaging differential diagnosis includes bacterial osteomyelitis, sarcoma and bone cysts, among other pathologies.

In ultrasound, the presence of hypoechoic lesions containing small hyperechoic foci is considered characteristic of mycetoma; they are associated with the 
presence of grains, capsule and peripheral inflammation. In general, they are better visualized in eumycetoma than in actinomycetoma, since the latter usually has less fistulae, which hamper hyper-reflective echoes' signal ${ }^{38}$.

Magnetic resonance is a non-invasive technique that facilitates soft tissue and bone destruction visualization, and it is therefore useful to assess the mycetoma extent. Grains appear as round-shaped, low-intensity, small lesions of 2 to $5 \mathrm{~mm}$ in diameter (which remind of a dot), in clusters surrounded by a high-intensity ring (resembling a circle) that represents the peripheral granulomatous reaction. This characteristic image is known as the "dot in a circle sign"39-42, and it was subsequently recognized in ultrasound-obtained images, where it is observed as a hyperechoic area (dot) surrounded by hypoechoic tissue (circle) $)^{43,44}$. El Shamy et al. proposed a scale to assess mycetoma seriousness using magnetic resonance findings. The degree of compromise is assessed in three planes: skin, muscle and bone. Cutaneous and subcutaneous findings include skin destruction, abscess formation and fistulae with or without presence of grains. In muscle, edema and microabscesses and macroabscesses formation can be observed. In bones, it is possible for bone marrow edema, cavitation and bone destruction to be found. Lesions are classified as mild, moderate or severe according to the sum of the scores obtained for each plane ${ }^{45}$.

\section{Molecular diagnosis}

Culture and molecular techniques are not generally available in endemic areas; however, the treatment of eumycetoma can vary depending on the causative agent, and sometime, exact identification only can be achieved with molecular techniques. The tools used include amplification by polymerase chain reaction (PCR), followed by sequencing of the region of interest such as, for example, the region comprising the ribosomal genes and the intergenic regions (ITS $)^{46}$. PCR products can also be used for identification by other, less sensitive, but less expensive techniques, such as restriction fragment length polymorphism ${ }^{47}$. More recent versions, such as real-time PCR, have similarly been employed to identify eumycetoma-causative species using tissue samples or isolates of the environment ${ }^{48,49}$. Recently, two techniques were proposed, recombinase polymerase amplification (RPA) and loop-mediated isothermal amplification (LAMP), which are suggested as alternatives to phenotypical methods. Both methods demonstrated high specificity and sufficient sensitivity, and are appropriate for the detection of M. mycetomatis; however, RPA is simpler and has higher potential to be implemented in endemic areas $^{50}$.

Other genes used for eumycetoma-causative fungal species include beta tubulin, nuclear ribosomal RNA large and small subunits (nucLSU and nucSSU, respectively), RNA polymerase second subunit in size and elongation factor alpha ${ }^{51}$.

\section{Advances in molecular identification and phylogeny}

The use of molecular diagnostic in phylogeny and taxonomy revealed errors in the classification of isolates previously identified with traditional methods. One recent phylogenetic analysis demonstrated that $M$. mycetomatis and $M$. grisea belong to different orders; currently, the Madurella genus is comprised by M. mycetomatis, $M$. pseudomycetomatis, $M$. tropica and M. fahalii, which belong to the Sordiales order, whereas M. grisea is member of the Pleosporales and went on to be denominated Trematosphaeria grisea ${ }^{29,30,52,53}$. In addition, four CBS sterile mycelium dematiaceous strains obtained from patients with mycetoma, originally identified by traditional methods as M. mycetomatis, showed significant differences in the ITS region, thus being demonstrated that they belong to different species; none of these strains was native to Africa ${ }^{28}$. Subsequently, inclusion of the Madurella genus in the Chaetomiaceae family was demonstrated; member of this family are commonly found in dung and manure, which drove to conclude that the manure present in endemic areas can be a niche for Madurella and play an essential role in eumycetoma acquisition ${ }^{54}$.

Molecular identification is also used to infer ecological data based on the phylogenetic relationships with species for which there is more information available. Most strains obtained from human eumycetomas were found to correspond to species that are related to marine fungi, which suggests an association between virulence and halotolerance ${ }^{31}$.

\section{Auxiliary methods}

Immunologic methods, such as enzyme-linked immunosorbent assay (ELISA), which detect the presence of specific antibodies, are regarded as; however, they are used for epidemiological studies rather than for clinical diagnosis. 
Other tools to identify the causative agent include immunohistochemistry and matrix assisted laser desorption ionization time-of-light mass-spectrometry (MALDI-TOF); however, the complexity and costs of these techniques limit their availability ${ }^{55,56}$.

\section{Differential diagnosis}

Many infectious and non-infectious diseases can be mixed up with eumycetoma. The first difference should be established with actinomycetomas, which in general terms are more aggressive, produce more inflammation and fistulae, dissemination to lymphatic vessels is common, and bone compromise is early, but with small cavities. In turn, eumycetomas produce few fistulae, bone compromise is usually late and with large cavities, and lymphatic dissemination is occasional; on microscopic examination, the lesion is well defined and has capsule 1 . However, clinical examination is not sufficient to establish a distinction between these pathologies, which is why the microorganism should be isolated in order to confirm the mycetoma etiology. Among infectious diseases, cutaneous tuberculosis, atypical bacteria infections with atypical bacteria, chromomycosis, sporotrichosis, cutaneous blastomycosis, leishmaniasis, hyalohyphomycosis, botryomycosis and cellulitis should be considered as differential diagnoses. Eumycetoma initial lesions can also be confused with foreign body granulomas and different neoplasms, both benign and malignant ${ }^{6}$.

\section{Treatment}

Eumycetomas treatment includes debridement of affected tissues plus antifungal therapy, usually for one year, which can be extended to 18-24 months. Azoles are the most widely used drugs, owing to their availability and low cost in comparison with other antifungals. Itraconazole (400 mg/day) is considered first-choice, whereas ketoconazole (400-800 mg/day) is less used due to its hepatotoxicity. However, high treatment-resistance rates are reported, with recurrence ranging from $20 \%$ to $90 \%{ }^{57}$. This might be related to identification, by traditional methods, of different species that possess different antifungal resistance profiles, such as M. mycetomatis. For example, M. fahalii (CBS 129176) showed resistance to 5 -flucytosine, fluconazole, itraconazole and caspofungin, and $M$. tropica (CBS 201.38) was resistant to 5-fluorocytosine and caspofungin ${ }^{29,30}$. The use of broad-spectrum strong triazoles, such as posaconazole $(200-800 \mathrm{mg} /$ day) and voriconazole, yields high rates of cure (80\%) against Madurella and $S$. apiospermum, with remission being observed during follow-up 2 years after treatment conclusion, but its high cost precludes its use in endemic regions ${ }^{58-61}$. According to Dupont et al., in $20 \%$ of resistance cases diclofenac (100 mg/day) can be an efficacious concomitant treatment, they report a eumycetoma case produced by a multi-drug resistant $M$. mycetomatis, where adition of non-steroidal anti-inflammatory drug (NNSAID) was associated with clinical, radiological and functional improvement. As regards this clinical observation, we found that in vitro and in vivo studies demonstrated that other NSAIDs, such as ibuprofen, potentiate the azoles fungistatic activity; although the mechanism of action is not known to this moment, it is believed that it might be associated with ibuprofen inhibitor effect on ATP-dependent transport pumps, which are related to fungal resistance ${ }^{64}$. On the other hand, studies in Candida albicans demonstrated that NSAIDs reduce the formation of biofilm, which favors drug access to the fungus ${ }^{66}$. In addition, in bacterial-origin osteomyelitis, NSAIDs decrease bacterial cytotoxic effect on osteoblasts ${ }^{66}$.

With regard to the use of other antifungals, in vitro, terbinafine alone or in combination with ketoconazole or itraconazole showed no synergistic effect or better response than azoles in the treatment of eumycetomas caused by $M$. mycetomatis, and in infections with S. apiospermium and $S$. boydii, terbinafine showed no benefits, and its use is therefore not indicated, at least in most eumuycetomas ${ }^{67}$. In disseminated cases or which don't respond to initial treatment, intravenous amphotericin B is used at a total dose of 2 to $4 \mathrm{~g}$, alone or combined with voriconazole or micafungin ${ }^{68}$.

In conclusion, the best treatment for eumycetoma includes antifungals plus surgery; these drugs are indicated prior and after surgical excision. During surgery, subcutaneous nodules must be eliminated intact to prevent dissemination of the grains to the adjacent tissue ${ }^{69}$. The prolonged duration of treatment, together with the low socioeconomic stratus most patients belong to the low income group of most patients, favors therapeutic schemes non-compliance, which increses resistance, and is reflected in higher likelihood of complications and radical surgical treatments, such as amputation ${ }^{1,2}$.

\section{Experimental models}

To develop better therapeutic strategies, study models mimicking the infectious agent status inside the 
patient are required. Similarly to other fungal agents, eumycetoma-causative fungi produce a protective structure that surrounds the hypha. The presence of grains is key in eumycetoma, but their formation and sensitivity to antifungals is not yet fully understood. Partially, this is so because grains cannot be induced in vitro, with a mammal host being required to elicit their formation. In the past, mice and monkeys were used to stimulate the formation of grains simulating mycetomas by $M$. mycetomatis, with large inoculum concentrations being required and with variable rates of success and reproducibility difficulties for other authors. When the grain was obtained in animal models, it resembled the one formed in humans, characterized by mycelia embedded in a cement-like material surrounded by neutrophils ${ }^{70}$. Given that both in humans and in animal models there are neutrophils found surrounding the grain, these cells are considered to be important in its formation. However, neutrophils cannot currently be regarded as the only effector cell involved, given that exposure of $M$. mycetomatis to neutrophils does not elicit the formation of grain, that were development in vitro. As an alternative to induce grain formation, the larva of the greater wax moth (Galleria mellonella) was used; the grains produced in this larva resemble those found in humans and mammals, and this infection model might be used in the study of grain formation and therapeutic responses to antifungal agents in $M$. mycetomatis ${ }^{3}$.

Finally, other fungi described in eumycetomas, such as Aspergillus, Scedosporium and Fusarium species, also recognized as opportunistic infections causative agents, were studied using Drosophila melanogaster as host, and the treatment with voriconazole was found to protect the flies against the infection, except in those infected with Scedosporium prolificans, which showed no improvement ${ }^{71,72}$.

\section{Conclusions}

Although eumycetoma has a low prevalence in Europe and Latin America, knowledge about this mycosis is essential, since ignorance on the subject leads to diagnostic delay and inadequate patient management, which constitutes a health problem, especially in rural areas. On the other hand, the current process of human migration from Africa to Europe will probably increase the presence of this mycosis in the Old Continent, and it should therefore be considered among the diagnostic possibilities. Correct identification of the causative agent is imperative in order for In order to start appropriate treatment as soon as possible. Currently, molecular biology techniques, such as PCR and sequencing, enable correct identification when traditional methods fail. Given the presence of resistant strains against first-line therapeutic agents, it is important for antifungal sensitivity tests to be carried out.

\section{Conflicts of interests}

None to be declared.

\section{References}

1. Zijlstra EE, van de Sande WW, Welsh O, et al. Mycetoma: a unique neglected tropical disease. Lancet Infect Dis. 2016;16:100-12.

2. Fahal A, Mahgoub ES, El Hasan AM, et al. A new model for management of mycetoma in the Sudan. PloS Negl Trop Dis. 2014;8:e3271.

3. Kloezen W, van Helvert-van Poppel M, Fahal AH, et al. A Madurella mycetomatis grain model in Galleria mellonella larvae. Plos Negl Trop Dis. 2015;9:e0003926.

4. Estrada R, Chávez-Lopez G, Estrada-Chavez G, et al. Eumycetoma. Clin Dermatol. 2012;30:389-396.

5. Rao KV, Praveen A, Megha S, et al. Atypical craniocerebral eumycetoma: a case report and review of literature. Asian J Neurosurg. 2015; 10:56-9.

6. Nenoff $\mathrm{P}$, van de Sande WW, Fahal $\mathrm{AH}$, et al. Eumycetoma and actinomycetoma - an update on causative agents, epidemiology, pathogenesis, diagnostics and therapy. J Eur Acad Dermatol Venereol. 2015; 29:1873-83.

7. Samy AM, van de Sande WWJ, Fahal AH, et al. Mapping the potential risk of Mycetoma infection in Sudan and South Sudan using ecological niche modeling. Plos Negl Trop Dis. 2014;8:e3250.

8. Khatri ML, Al-Halali HM, Fouad-Khalid M, et al. Mycetoma in Yemen: clinicoepidemiologic and histopathologic study. Int J Dermatol. 2002; 41:586-93.

9. Padin C, Fernández-Zeppenfeldt G, Yegres F, et al. Scytalidium dimidiatum: hongo oportunista para el hombre y árboles de Mangifera indica en Venezuela. Rev Iberoam Micol. 2005;22:172-3.

10. Queiroz-Telles F, Nucci M, Lopes-Colombo A, et al. Mycoses of implantation in Latin America: an overview of epidemiology, clinical manifestations diagnosis and treatment. Med Myc. 2011:49:225-36.

11. Schwartz E, Shapiro A. Madura foot or philoctetes foot? Isr Med Assoc J. 2015;17:442-4.

12. Bonifaz A, Tirado-Sánchez A, Calderón L, et al. Mycetoma: experience of 482 cases in a single center in Mexico. PloS Negl Trop Dis. 2014; 8:e3102.

13. López-Martínez R, Méndez-Tovar L, Bonifaz A, et al. Actualización de la epidemiología del micetoma en México. Revisión de 3,933 casos. Gac Med Mex. 2013;149:586-92.

14. Bounfrate D, Gobbi F, Angheben A, et al. Autochthonous cases of mycetoma in Europe: report of two cases an review of literature. PloS One. 2014:9:e100590

15. Brufman T, Ben-Ami R, Mizrahi M, et al. Mycetoma of the foot caused by Madurella mycetomatis in immigrants from Sudan. Isr Med Assoc J. 2015;17:418-20.

16. Elad D, Blum S, Kol A, et al. Eumycetoma caused by Madurella mycetomatis in a mare. Med Mycol. 2010:48:639-42.

17. Ahmed M, Sureka J, Chacko G, et al. MRI findings in cranial eumycetoma. Indian J Radiol Imagging. 2011;21:261-3.

18. Elhardello OA, Adam ES, Adam I. Abdominal wall mycetoma presented as obstructed incisional hernia of cesarean section in Eastern Sudan. Infect Dis Obst Gynec. 2007;2007:74643.

19. Mirza SH, Gardezi AH, Khan Y, et al. Subcutaneous facial mycosis in a child due to Madurella mycetomatis. J Pak Med Assoc. 2007:57:466-8.

20. El Amrani F. Le pied de Madura. Pan Afr Med J. 2013;14:24.

21. Sampaio FM, Galhardo MC, de Farias-Cardoso R, et al. Eumicetoma on the foot caused by Madurella mycetomatis: amputation after significant worsening during pregnancy. Acta Derm Venereol. 2015;95:374-5.

22. Ahmed A, Adelmann D, Fahal A, et al. Environmental occurrence of Madurella mycetomatis, the major agent of human eumycetoma in Sudan. J Clin Microbiol. 2002:40:1031-6.

23. Verwer PE, Notenboom CC, Eadie K, et al. A polymorphism in the chitotriosidase gene associated with risk of mycetoma due to Madurella 
mycetomatis. Mycetoma - a retrospective study. Plos Negl Trop Dis. 2015;9:e0004061.

24. Daoud M, Ezzine Sebai N, Badri T, et al. Mycetoma: retrospective study of 13 cases in Tunisia. Acta Dermatovenerol Alp Pannoica Adriat. 2005;14:153-6.

25. Kondori N, Baltzer L, Dolphin GT, et al. Fungicidal activity of human lactoferrin-derived peptides based on the antimicrobial $\alpha \beta$ region. Int $J$ Antimicrob Agents. 2011;37:51-7.

26. Van de Sande WW, Fahal A, Verbrugh $\mathrm{H}$, et al. Polymorphisms in genes involved in innate immunity predispose toward mycetoma susceptibility. $\mathrm{J}$ Immunol. 2007;179:3065-74.

27. Nasr A, Abushouk A, Hamza A, et al. Th-1, Th-2 cytokines profile among Madurella mycetomatis eumycetoma patients. PloS Negl Trop Dis. 2016;10:e0004862.

28. Hijra N, Boudhas A, Al Bouzidi A, et al. Madura foot: report of a eumycetoma Moroccan case. J Dermtol Surg. 2015;19:143-5.

29. de Hoog GS, Adelmann D, Ahmed AO, et al. Phylogeny and typification of Madurrella mycetomatis, with a comparison of other agents of eumycetoma. Mycoses. 2004:47:121-30.

30. de Hoog GS, van Diepeningen AD, Mahgoub ES, et al. New species of Madurella, causative agents of black grain mycetoma. J Clin Microbiol. 2012;50:988-94.

31. Ahmed SA, van de Sande WWJ, Stevens DA, et al. Revision of agents of black-grain eumycetoma in the order Pleosporales. Persoonia. 2014;33:141-54.

32. Cortez K, Roilides E, Quiroz-Telles F, et al. Infections caused by Scedosporium spp. Clin Microb Rev. 2008;21:157-97.

33. Gilgado F, Gené J, Cano J, et al. Heterothalism in Scedosporium apiospermum and description of its teleomorph Pseudollescheria apiosperma sp. Nov. Med Mycol. 2010;48:122-8.

34. Gulati V, Bakare S, Tibrewal S, et al. A rare presentation of concurrent Scedosporium apiospermum and Madurella grisea eumycetoma in an immunocompetent host. Case Rep Pathol. 2012;2012:154201.

35. Ibrahim Al, El Hassan AM, Fahal A, et al. A histopathological exploration of the Madurella mycetomatis grain. PloS One. 2013;8:e57774.

36. Asly M, Rafaoui A, Bouyermane $\mathrm{H}$, et al. Mycetoma (Madura foot): a case report. Ann Phys Rehab Med. 2010;53:650-4.

37. Abd El Bagi ME. New radiographic classification of bone involvement in pedal mycetoma. AJR Am J Roentgenol. 2003;180:665-8.

38. Van de Sande WWJ, Fahal AH, Goodfellow M, et al. Merits and pitfalls of currently used diagnostic tools in mycetoma. Plos Negl Trop Dis. 2014;8:e2918.

39. Cherian RS, Betty M, Manipadam MT, et al. The dot-in-circle sign - a characteristic MRI finding in mycetoma foot: a report of three cases. $\mathrm{Br}$ J Radiol. 2009;82:662-5.

40. Jain V, Makwana GE, Bahri N, et al. The "dot in circle" sign on MRI in maduramycosis: a characteristic finding. J Clin Imaging Sci. 2012;2:66.

41. Kumar J, Kumar A, Sethy P, et al. The dot-in-circle sign of mycetoma on MRI. Diagn Interv Radiol. 2007;13:193-5.

42. Sarris I, Berendt AR, Athanasous N, et al. MRI of mycetoma of the foot: two cases demonstrating the dot-in-circle sign. Skeletal Radiol. 2003;32:179-83.

43. Laohawiriyakamol T, Tanutit $P$, Kanjanapradit K, et al. The "dot-in-circle" sign in musculoskeletal mycetoma on magnetic resonance imaging and ultrasonography. SpringerPlus. 2014;13:671.

44. Parker L, Singh D, Biz C. The dot-in-circle sign in Madura foot. J Foot Ank Surg. 2009;48:690.e1-5.

45. El Shamy ME, Fahal AH, Shakir MY, et al. New MRI grading system for the diagnosis and management of mycetoma. Trans R Soc Trop Med Hyg. 2012;106:738-42.

46. Mestre T, Vierira R, Coutinho J. Mycetoma of the foot - diagnosis of the etiologic agent and surgical treatment. Am J Trop Med Hyg. 2015;93:1-2.

47. Ahmed AA, Mukhtar M, Kools-Sijmons M, et al. Development of a species-specific PCR-restriction fragment length polymorphism analysis procedure for identification of Madurella mycetomatis. J Clin Microbiol. 1999;37:3175-8.

48. Ahmed AO, Desplaces N, Leonard P, et al. Molecular detection and identification of agents of eumycetoma: detail report of two cases. J Clin Microbiol. 2003;41:5813-6.

49. Year $H$, Bougnoux ME, Jeanrot $C$, et al. Mycetoma of the foot caused by Fusarium solani: identification of the etiological agent by DNA sequencing. J Clin Microbiol. 2003;41:1805-8.
50. Ahmed SA, van de Sande WW, Desnos-Ollivier M, et al. Application of isothermal amplification techniques for identification of Madurella mycetomatis, the prevalent agent of human mycetoma. J Clin Microbiol. 2015; 53:3280-5.

51. El Muttardi N, Kulendre D, Jemec B. Madura foot - mind the soil. J Plas Reconstr Aesthet Surg. 2010;63:e576-8

52. Van de Sande WWJ. Phylogenetic analysis of the complete mitochondrial genome of Madurella mycetomatis confirms its taxonomic position within the order Sordariales. PloS One. 2012;7:e38654

53. Yan J, Deng J, Zhou CJ, et al. Phenotypic and molecular characterization of Madurella pseudomycetomatis sp. Nov., a novel opportunistic fungus possibly causing black-grain mycetoma. J Clin Microbiol. 2010;48:251-7.

54. de Hoog GS, Ahmed SA, Najafzadeh MJ, et al. Phylogenetic findings suggest possible new habitat and routes of infection of human eumyctoma. PloS Negl Trop Dis. 2013;7:e2229.

55. Ahmed SA, Abbas MA, Jouvion G, et al. Seventeen years of subcutaneous infection by Aspergillus flavus; eumycetoma confirmed by immunohistochemistry. Mycoses. 2015;58:728-34.

56. Coulibaly $\mathrm{O}$, Marinach-Patrice $\mathrm{C}$, Cassagne $\mathrm{C}$, et al. Pseudallescheria/ Scedosporium complex species identification by matrix-assisted laser desorption ionization time-of-flight mass spectrometry. Med Myc. 2011;49:621-6.

57. Ameen A, Arenas R. Developments in the management of mycetomas. Clin Exp Dermatol. 2009;34:1-7.

58. Crabol Y, Poiree S, Bougnoux ME, et al. Last generation triazoles for imported eumycetoma in eleven consecutives adults. Plos Negl Ttop Dis. 2014;8:e3232.

59. Lacroix C, De Kerviler E, Morel P, et al. Madurella mycetomatis mycetoma treated successfully with oral voriconazole. Br J Dermatol. 2005 152:1067-8.

60. Loulergue P, Hot A, Dannaoui E, et al. Successful treatment of blackgrain mycetoma with voriconazole. Am J Trop Med Hyg. 2006;75:1106-7.

61. Sharma A, Sharma N, Nat A, et al. Case report: non-invasive management of Madura foot with oral posaconazole and ciprofloxacin. Am J trop Med Hyg. 2014;91:1259-62.

62. Dupont A. Non-steroidal anti-inflammatory drug to treat a eumycetoma. Lancet. 2016;16:779.

63. Dupont B, Datry A, Poirée S, et al. Role of a NSAID in the apparent cure of a fungal mycetoma. J Mycol Med. 2016;26:86-93.

64. Costa-de-Oliveira S, Miranda IM, Silva-Dias A, et al. Ibuprofen potentiates the in vivo antifungal activity of fluconazole against Candida albicans murine infection. Antimicrob Agents Chemother. 2015;59:4289-92.

65. Alen MAS, Douglas LJ. Effects of aspirin and other nonsteroidal anti-inflammatory drugs on biofilms and planktonic cells of Candida albicans. Antimicrob Agents Chemother. 2004;48:41-4.

66. Hendrix AS, Spoonmore TJ, Wilde AD, et al. Repurposing the nonsteroidal anti-inflammatory drug diflunisal as an osteoprotective, antivirulence therapy for Staphylococcus aureus osteomyelitis. Antimicrob Agents Chemother. 2016;60:5322-30.

67. Ahmed SA, Kloenzen W, Fahal A et al. In vitro interaction of currently used azoles with terbinafine against Madurella mycetomatis. Antimicrob Agents Chemother. 2015;59:1373-4

68. Rodríguez MM, Calvo E, Serena C, et al. Effects of double and triple combinations of antifungal drugs in a murine model of disseminated infection by Scedosporium prolificans. Antimicrob Agents Chemother. 2009;53:2153-5.

69. Soeiro-Sampaio FM, Pereira-Quintella L, Chicario de Oliveira Coelho JM, et al. Eumycetoma by Madurella mycetomatis with 30 years of evolution: therapeutic challenge. An Bras Dermatol. 2013;88(6 Suppl 1):S82-4.

70. Ahmed A, van Vianen W, ten Kate M, et al. A murine model of Madurrella mycetomatis eumycetoma. FEMS Immunol Med Microbiol. 2003;37:29-36.

71. Lamaris G, Chamilos G, Lewis RE, et al. Virulence studies of Scedosporium and Fusarium species in Drosophila melanogaster. $J$ Infect Dis. 2007;196:1860-4.

72. Lionakis MS, Lewis RE, May GS. Toll-deficient Drosophila flies as a fast, high-throughput model for the study of antifungal drug efficacy against invasive aspergillosis and Aspergillus virulence. J Infect Dis. 2005; 191:1188-95. 